\title{
Discrete-Time Negative Imaginary Systems
}

DOI:

10.1016/j.automatica.2017.01.001

\section{Document Version}

Accepted author manuscript

Link to publication record in Manchester Research Explorer

\section{Citation for published version (APA):}

Ferrante, A., Lanzon, A., \& Ntogramatzidis, L. (2017). Discrete-Time Negative Imaginary Systems. Automatica, 79, 1-10. https://doi.org/10.1016/j.automatica.2017.01.001

\section{Published in:}

Automatica

\section{Citing this paper}

Please note that where the full-text provided on Manchester Research Explorer is the Author Accepted Manuscript or Proof version this may differ from the final Published version. If citing, it is advised that you check and use the publisher's definitive version.

\section{General rights}

Copyright and moral rights for the publications made accessible in the Research Explorer are retained by the authors and/or other copyright owners and it is a condition of accessing publications that users recognise and abide by the legal requirements associated with these rights.

\section{Takedown policy}

If you believe that this document breaches copyright please refer to the University of Manchester's Takedown Procedures [http://man.ac.uk/04Y6Bo] or contact uml.scholarlycommunications@manchester.ac.uk providing relevant details, so we can investigate your claim.

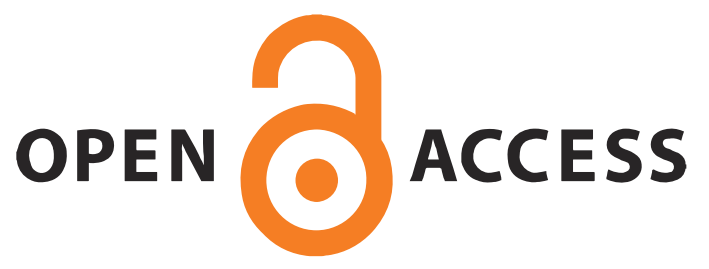




\title{
Discrete-Time Negative Imaginary Systems ${ }^{\star}$
}

\author{
Augusto Ferrante $^{\mathrm{a}}$, Alexander Lanzon ${ }^{\mathrm{b}}$, Lorenzo Ntogramatzidis $^{\mathrm{c}}$ \\ a Dipartimento di Ingegneria dell'Informazione, Università di Padova, via Gradenigo 6/B, Padova, Italy. \\ ${ }^{\mathrm{b}}$ School of Electrical and Electronic Engineering, University of Manchester, Sackville Street, Manchester M13 9PL, UK. \\ ${ }^{\mathrm{c} D e p a r t m e n t ~ o f ~ M a t h e m a t i c s ~ a n d ~ S t a t i s t i c s, ~ C u r t i n ~ U n i v e r s i t y, ~ P e r t h ~(W A), ~ A u s t r a l i a . ~}$
}

\begin{abstract}
In this paper we introduce the notion of a discrete-time negative imaginary system and we investigate its relations with discrete-time positive real system theory. In the framework presented here, discrete-time negative imaginary systems are defined in terms of a sign condition that must be satisfied in a domain of analyticity of the transfer function, in analogy with the case of discrete-time positive real functions, as well as analogously to the continuous-time case. This means in particular that we do not need to restrict our notions and definitions to systems with rational transfer functions. We also provide a discrete-time counterpart of the different notions that have appeared so far in the literature within the framework of strictly positive real and in the more recent theory of strictly negative imaginary systems, and to show how these notions are characterized and linked to each other. Stability analysis results for the feedback interconnection of discrete-time negative imaginary systems are also derived.
\end{abstract}

Key words: Discrete-time negative imaginary systems, discrete-time negative imaginary lemma, discrete-time positive real systems.

\section{Introduction}

The theory of positive real (PR) systems dates back to the early 1930s [5], and is regarded as one of the cornerstones of systems and control theory, and in particular of passivity theory. For a summary of the historic and recent contributions in this area, we refer the reader to the important monographs [1], [4]. A promising new development in the area has been the introduction of the notion of negative imaginary (NI) systems, see $[12,29,15]$ and the references therein. The definition of negative imaginary systems imposes a weaker restriction on the relative degree of the transfer function with respect to the one for positive real systems, and does not prohibit the case of all unstable transmission zeros. In the past few years, a rich stream of literature flourished on negative imaginary systems, including extensions

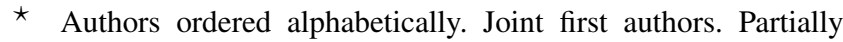
supported by the Australian Research Council under the grant FT120100604. Corresponding author L. Ntogramatzidis. Tel. +618-92663143. Fax +61-8-92663197.

Email addresses: augusto@dei.unipd.it (Augusto

Ferrante), Alexander.Lanzon@manchester.ac.uk (Alexander Lanzon), L.Ntogramatzidis@curtin.edu.au (Lorenzo Ntogramatzidis).
}

to infinite dimensional systems [18], Hamiltonian systems [25], descriptor systems [14], lossless negative imaginary systems [30] and mixtures of negative imaginary and smallgain properties [19] to mention only a few. The theory developed in these contributions has been proved to be useful in a range of applications including modelling and control of undamped or lightly damped flexible structures with colocated position sensors and force actuators [20,3], nanopositioning control due to piezoelectric transducers and capacitive sensors (e.g. $[2,17,16])$ and multi-agent networked systems (e.g. [6,26,27]). This theory provides a very general technique for finding an appropriate state-feedback controller and this is particularly useful when the underlying model is repeatedly derived by system identification techniques (see e.g. [31,33,32] and references therein).

An important gap in the current literature - that the present paper attempts to fill - is the lack of a definition of negative imaginary (and strictly negative imaginary) function for discrete-time systems. Furthermore, so far [9] and [8] have been the only contributions which attempted to address the general case of a definition of negative imaginary system for non necessarily rational transfer functions and then recover, in the symmetric rational case, the standard definition given in the foundational paper [12]. 
The main contribution of this paper is to introduce the notion of discrete-time negative imaginary systems for the first time. This definition is given in the general non-rational setting and then is specialized for rational transfer functions, and expressed in terms of a sign constraint on the unit circle. We also introduce different notions of strictly negative imaginary discrete-time transfer functions that parallel the continuous-time definitions given so far. Finally, the relations between discrete-time and continuous-time negative imaginary systems are elucidated. We also provide a discrete-time negative imaginary lemma which yields a complete state-space characterization of discrete-time negative imaginary systems and a stability analysis result for the feedback interconnection of discrete-time negative imaginary systems.

Notice that negative imaginary system theory has already been proven to be very useful in the continuos-time; hence developing a discrete-time counterpart of this theory is particularly significant and promising in view of the pervasive role of digital control in modern applications.

Notation. Given a matrix $A$, the symbol $A^{\top}$ denotes the transpose of $A$ and $A^{*}$ denotes the complex conjugate transpose of $A$. We denote by $\sigma(A)$ the set of singular values of the matrix $A$ and by $\underline{\sigma}(A)$ the smallest of such singular values. The usual notations of $\geq 0$ and $>0$ are used to denote positive semidefiniteness and positive definiteness of Hermitian matrices, respectively. Let $G: \mathbb{C} \longrightarrow \mathbb{C}^{m \times m}$ be analytic or harmonic in a certain region $\Omega$ of $\mathbb{C}$, then $G$ is said to have full normal rank if there exists $z \in \Omega$ such that $\operatorname{det}[G(z)] \neq 0$.

\section{Discrete-Time Positive Real Functions}

In this section, for the sake of completeness we briefly recall the most important notions and results of discrete-time positive real systems. The definition of discrete-time positive real function was introduced for the first time in the literature by Hitz and Anderson in [10], and is recalled below.

Definition 2.1 [10]. The function $F: \mathbb{C} \longrightarrow \mathbb{C}^{m \times m}$ is discrete-time positive real $(P R)$ if

- $F(z)$ is analytic in $\{z \in \mathbb{C}:|z|>1\}$;

- $F(z)$ is real when $z$ is real and positive;

- $F(z)^{*}+F(z) \geq 0$ for all $|z|>1$.

Similarly to what happens in the continuous-time for rational functions, discrete-time positive realness can be characterized in terms of conditions involving properties of the restriction of the matrix function to the unit circle.

Theorem 2.1 [10, Lemma 2]. Let $F: \mathbb{C} \longrightarrow \mathbb{C}^{m \times m}$ be a discrete-time, real, rational, proper transfer function. Then, $F(z)$ is $P R$ if and only if

- $F(z)$ has no poles in $\{z \in \mathbb{C}:|z|>1\}$;

- $F\left(e^{i \theta}\right)^{*}+F\left(e^{i \theta}\right) \geq 0$ for all $\theta \in[0,2 \pi)$ except for the values of $\theta$ for which $z=e^{i \theta}$ is a pole of $F(z)$;
- If $z_{0}=e^{i \theta_{0}}$, with $\theta_{0} \in[0,2 \pi)$, is a pole of $F(z)$, then it is a simple pole and the normalized residual matrix

$$
K_{0} \stackrel{\text { def }}{=} \frac{1}{z_{0}} \lim _{z \rightarrow z_{0}}\left(z-z_{0}\right) F(z)
$$

is Hermitian and positive semidefinite.

We now present a definition of discrete-time strictly positive real systems. We warn the reader that many different definitions have been proposed for this concept that can indeed be distinguished via several grades of strength, see e.g. $[4,11]$. In this paper, we shall only need two of such grades, that will be referred to as strongly and weakly strictly positive realness.

Definition 2.2 Let $F: \mathbb{C} \longrightarrow \mathbb{C}^{m \times m}$ be a discrete-time, real, proper transfer function. Then, $F(z)$ is discrete-time strongly strictly positive real $(S S P R)$ if for some $\delta \in(0,1)$, the transfer function $F(\delta z)$ is $P R$ and $F(z)+F(1 / z)^{\top}$ has full normal rank.

The following result shows that in the case of rational functions the property of SSPR is equivalent to an analyticity condition and a sign condition restricted to the unit circle.

Theorem 2.2 Let $F: \mathbb{C} \longrightarrow \mathbb{C}^{m \times m}$ be a discrete-time, real, rational, proper transfer function. Then, $F(z)$ is SSPR if and only if

- $F(z)$ has all its poles in a disk of radius $\rho \in[0,1)$;

- $F\left(e^{i \theta}\right)+F\left(e^{i \theta}\right)^{*}>0$ for all $\theta \in[0,2 \pi)$.

Proof: Necessity of the first condition is obvious. Necessity of the second immediately follows from the fact the unit circle is in the interior of the domain of analyticity and by the full normal rank assumption. As for sufficiency, since the unit circle is closed, condition $F\left(e^{i \theta}\right)+F\left(e^{i \theta}\right)^{*}>0$ for all $\theta \in[0,2 \pi)$ implies coercivity, i.e. there exists $\sigma_{0}>0$ such that $F\left(e^{i \theta}\right)+F\left(e^{i \theta}\right)^{*}>\sigma_{0} I$ for all $\theta \in[0,2 \pi)$. Therefore, there exists $\rho \in[0,1)$ such that $F\left(\rho e^{i \theta}\right)+F\left(\rho e^{i \theta}\right)^{*}>0$ for all $\theta \in[0,2 \pi)$, so that $F_{1}(z) \stackrel{\text { def }}{=} F(\rho z)$ is PR.

Remark 2.1 The conditions of Theorem 2.2 are much simpler than those of its continuous-time counterpart (see e.g. [4, Theorem 2.47] and [11, Lemma 6.1]) because positivity on the unit circle $\mathbb{T}$ implies coercivity in view of the closure of the unit circle $\mathbb{T}$ (as opposed to the fact that the imaginary axis is not closed). As we shall see later, this is not the case for discrete-time negative imaginary (NI) systems for which the relevant boundary curve is the intersection between $\mathbb{T}$ and the open upper half complex plane. Therefore, the relevant boundary curve is not closed as the zero and infinity discrete frequencies are not in this curve. This fact complicates the derivations and the results in the NI case.

The next result is the discrete-time counterpart of the socalled positive real lemma, a cornerstone of modern control theory that has generated an endless stream of literature. 
Lemma 2.1 [Discrete-Time Positive Real Lemma, [10, Lemma 3]]. Let $F: \mathbb{C} \longrightarrow \mathbb{C}^{m \times m}$ be a discrete-time, real, rational, proper transfer function with no poles in $|z|>$ 1 and simple poles only on $|z|=1$. Let $\left[\frac{A \mid B}{C}|D|\right.$ be a minimal realization of $F(z)$. Then $F(z)$ is discrete-time positive real if and only if there exist a real matrix $X=X^{\top}>0$ and real matrices $L$ and $W$ such that

$$
\begin{aligned}
& X-A^{\top} X A=L^{\top} L, \\
& C^{\top}-A^{\top} X B=L^{\top} W, \\
& D^{\top}+D-B^{\top} X B=W^{\top} W .
\end{aligned}
$$

\section{Discrete-Time Negative Imaginary Functions}

We now introduce the following standing assumption that will be used throughout the rest of the paper.

Assumption 3.1 We henceforth restrict our attention to only symmetric transfer functions.

As discussed in [9], the case of symmetric transfer function is the most important one, because it encompasses both the scalar case, and the case of a transfer function of a reciprocal $m$-port electrical network. ${ }^{1}$ To the best of our knowledge, all the negative imaginary transfer functions considered or studied in the literature so far are symmetric (see e.g. the transfer functions from a force actuator to a corresponding collocated position sensor - for instance, a piezoelectric sensor - in a lightly damped or undamped structure), even though the real, rational definitions of negative imaginary systems in $[12,29,15]$ allow for non-symmetric transfer functions.

We now present a definition of negative imaginary functions in the discrete-time case.

Definition 3.1 Let $G: \mathbb{C} \longrightarrow \mathbb{C}^{m \times m}$ be a discrete-time, real transfer function. We say that $G(z)$ is discrete-time negative imaginary $(N I)$ if

(i) $G(z)$ is analytic in $\{z \in \mathbb{C}:|z|>1\}$;

(ii) $i\left[G(z)-G(z)^{*}\right] \geq 0$ for all $z \in \mathbb{C}$ such that $|z|>1$ and $\mathfrak{I m}(z)>0$

(iii) $i\left[G(z)-G(z)^{*}\right]=0$ for all $z \in \mathbb{C}$ such that $|z|>1$ and $\mathfrak{I m}(z)=0$

(iv) $i\left[G(z)-G(z)^{*}\right] \leq 0$ for all $z \in \mathbb{C}$ such that $|z|>1$ and $\mathfrak{I m}(z)<0$.

\footnotetext{
1 We recall that the only way to obtain a non-symmetric transfer function of an $m$-port electrical network is to employ gyrators, whose physical implementation requires the use of active components but that cannot be physically implemented with arbitrary precision.
}

The conditions (ii)-(iv) in Definition 3.1 are a skew imaginary condition on the open set $\Omega=\{z \in \mathbb{C}:|z|>1\}$.

Remark 3.1 Note that if the real transfer function $G: \mathbb{C} \longrightarrow$ $\mathbb{C}^{m \times m}$ satisfies the conditions in Definition 3.1, then $G(z)$ is symmetric, i.e., $G(z)=G(z)^{\top}$ for all $z \in \mathbb{C}$ such that $|z|>1$. This can be seen as follows: since $G(z)$ is real, if $z \in \mathbb{R}$ then $G(z) \in \mathbb{R}$. Let $z \in \mathbb{R}$ and $|z|>1$. From (iii), we get $G(z)=G(z)^{\top}$. Therefore, each entry $\Delta_{i j}(z)$ of the matrix valued function $\Delta(z) \stackrel{\text { def }}{=} G(z)-G(z)^{\top}$ is a function which is analytic in $\{z \in \mathbb{C}:|z|>1\}$ and is zero for any real $z$ in the domain of analyticity. Then, in view of the principle of identity of analytic functions (see e.g. Corollary to Theorem 10.18 in [22, page 209]) $\Delta_{i j}(z)=0$ in the entire domain of analyticity so that $G(z)=G(z)^{\top}$ for all $z$ in the domain of analyticity, i.e., $|z|>1$.

Conditions (iii)-(iv) in Definition 3.1 are redundant in the rational case, as the following result establishes.

Lemma 3.1 Let $G: \mathbb{C} \longrightarrow \mathbb{C}^{m \times m}$ be a discrete-time, real, rational transfer function. If $G(z)$ satisfies (i)-(ii) of Definition 3.1, then it also satisfies (iii)-(iv).

Proof: If $G(z)$ satisfies (ii), then $i\left[G(z)^{\top}-G(\bar{z})\right] \geq 0$ for all $z \in \mathbb{C}$ such that $|z|>1$ and $\mathfrak{I m}(z)>0$, since $G(z)^{*}=$ $G(\bar{z})^{\top}$. Defining $w \stackrel{\text { def }}{=} \bar{z}$, such condition can be re-written as $i\left[G(w)^{*}-G(w)\right] \geq 0$ for all $w \in \mathbb{C}$ such that $|w|>1$ and $\mathfrak{I m}(w)<0$, which is exactly (iv) of Definition 3.1. Finally, since (ii) and (iv) hold, then (iii) must also hold by continuity.

We now prove the counterpart of Theorem 2.1 for the case of discrete-time symmetric negative imaginary functions. This result provides a characterization of rational NI systems in terms of a domain of analyticity and conditions referred to the unit circle. First, however, we recall that given a real rational function $G(z)$ and a simple pole $p \in \mathbb{C}$ of $G(z)$, we have a unique decomposition $G(z)=G_{1}(z)+A /(z-p)$, where $G_{1}(z)$ is a rational function which is analytic in an open set containing $p$ and the (non-zero) matrix $A$ is the residue corresponding to the pole $p$. If $p$ is a double pole of $G(z)$, we have the unique decomposition $G(z)=G_{1}(z)+$ $A_{1} /(z-p)+A_{2} /(z-p)^{2}$, where the matrix $A_{1}$ is the residue corresponding to the pole $p$. In this case, by analogy, we define the (non-zero) matrix $A_{2}$ to be the quadratic residue corresponding to the pole $p$. If $G(z)$ has a pole at infinity, it can be uniquely decomposed as $G(z)=G_{1}(z)+P(z)$, where $G_{1}(z)$ is a rational proper function and $P(z)=\sum_{i=1}^{k} A_{i} z^{i}$ is a homogeneous polynomial in $z$. We refer to $A_{i}$ as the $i$-th coefficient in the expansion at infinity of $G(z)$.

Lemma 3.2 Let $G: \mathbb{C} \longrightarrow \mathbb{C}^{m \times m}$ be a discrete-time, real, rational, proper transfer function. Then, $G(z)$ is NI if and only if

(i) $G(z)$ has no poles in $|z|>1$; 
(ii) $i\left[G\left(e^{i \theta}\right)-G\left(e^{i \theta}\right)^{*}\right] \geq 0$ for all $\theta \in(0, \pi)$ except for the values of $\theta$ for which $z=e^{i \theta}$ is a pole of $G(z)$;

(iii) if $z_{0}=e^{i \theta_{0}}$, with $\theta_{0} \in(0, \pi)$, is a pole of $G(z)$, then it is a simple pole and the normalized residual matrix

$$
K_{0} \stackrel{\text { def }}{=} \frac{1}{z_{0}} \lim _{z \rightarrow z_{0}}\left(z-z_{0}\right) i G(z)
$$

is Hermitian and positive semidefinite;

(iv) if $z_{0}=1$ is a pole of $G(z)$, then it is at most a double pole. Moreover, its residue $A_{1}$ and its quadratic residue $A_{2}$ (when the pole is simple it is assumed that $A_{2}=0$ ) are Hermitian matrices satisfying $A_{2} \geq 0$ and $A_{1} \geq A_{2}$;

(v) if $z_{0}=-1$ is a pole of $G(z)$, then it is at most a double pole. Moreover, its residue $A_{1}$ and its quadratic residue $A_{2}$ (when the pole is simple it is assumed that $A_{2}=0$ ) are Hermitian matrices satisfying $A_{2} \leq 0$ and $A_{1} \geq$ $-A_{2}$.

Proof: The idea of the proof is the following: we introduce a bilinear transform and show that it maps continuous-time NI systems into discrete-time NI systems. Then we show that the conditions of Lemma 3.2 are mapped by the bilinear transform into the necessary and sufficient conditions derived in [9, Lemma 3.1] for a continuous-time system to be NI. Let $G(z)$ be discrete-time real, symmetric and rational, and define

$$
G_{c}(s) \stackrel{\text { def }}{=} G\left(\frac{1+s}{1-s}\right) .
$$

Consider the bilinear transform

$$
z=\frac{1+s}{1-s}
$$

and let $z=\sigma+i \omega$. It is found that

$s=\frac{z-1}{z+1}=\frac{\sigma^{2}+\omega^{2}-1}{(\sigma+1)^{2}+\omega^{2}}+2 i \frac{\omega}{(\sigma+1)^{2}+\omega^{2}}$.

Firstly, $G(z)$ is NI if and only if $G_{c}(s)$ is NI as a continuoustime transfer function. Indeed, in view of $(5), G(z)$ is analytic in $|z|>1$ if and only if $G_{c}(s)$ is analytic in $\mathfrak{R e}\{s\}>0$. The rest of the proof of this part follows directly from the definitions, using the fact that $\mathfrak{I m}\{z\}>0$ (resp. $\mathfrak{I m}\{z\}<0$ and $\mathfrak{I m}\{z\}=0$ ) is equivalent to $\omega>0$ (resp. $\omega<0$ and $\omega=0$ ), which in turn is equivalent to $\mathfrak{I m}\{s\}>0$ (resp. $\mathfrak{I m}\{s\}<0$ and $\mathfrak{I m}\{s\}=0$ ).

Secondly, the following facts are easy to check:

- $G(z)$ has no poles in $|z|>1$ if and only if $G_{c}(s)$ has no poles in $\mathfrak{R e}\{s\}>0$;

- Let $z_{0} \stackrel{\text { def }}{=} e^{i \theta_{0}}$ with $\theta_{0} \in(0, \pi)$. Using (5) we see that

$$
s_{0} \stackrel{\text { def }}{=} \frac{z_{0}-1}{z_{0}+1}=\frac{e^{j \theta_{0}}-1}{e^{j \theta_{0}}+1}=i \frac{\sin \theta_{0}}{1+\cos \theta_{0}},
$$

which shows that $z_{0}$ is a pole of $G(z)$ if and only if $i \omega_{0}$, with $\omega_{0} \stackrel{\text { def }}{=} \frac{\sin \theta_{0}}{1+\cos \theta_{0}}>0$, is a purely imaginary pole of $G_{c}(s)$. Moreover, $i\left[G\left(e^{i \theta}\right)-G\left(e^{i \theta}\right)^{*}\right] \geq 0$ for all $\theta \in$ $(0, \pi)$ such that $e^{i \theta}$ is not a pole of $G(z)$ if and only if $i\left[G_{c}(i \omega)-G_{c}(i \omega)^{*}\right] \geq 0$ for all $\omega \in(0, \infty)$ such that $i \omega$ is not a pole of $G(z)$;

- Let $z_{0} \stackrel{\text { def }}{=} e^{i \theta_{0}}$ with $\theta_{0} \in(0, \pi)$. Then $z_{0}$, with $\theta_{0} \in(0, \pi)$, is a pole of $G$ if and only if $i \omega_{0}$, with $\omega_{0} \stackrel{\text { def }}{=} \frac{\sin \theta_{0}}{1+\cos \theta_{0}}>0$, is a purely imaginary pole of $G_{c}$. Moreover, they are poles with the same multiplicity. Finally, $z_{0}$ is a simple pole of $G(z)$ with residue being the matrix $K$ if and only if $i \omega_{0}$ is a simple pole of $G_{c}(s)$ with residue being the matrix $H \stackrel{\text { def }}{=} \frac{e^{-i \theta_{0}}}{1+\cos \theta_{0}} K$. Notice that the normalized residual matrix $K_{0}$ of $G(z)$, as defined in (4), is positive semi-definite if and only if $\frac{i}{z_{0}} K$ is positive semi-definite and, hence, if and only if $i \stackrel{z_{0}}{H}$ is positive semi-definite.

- $z_{0}=1$ is a pole of $G(z)$ if and only if $s_{0}=0$ is a pole of $G_{c}$. This fact follows straightforwardly from (5). Moreover, they are poles with the same multiplicity. If this multiplicity is strictly greater than 2 , then $G(z)$ is trivially not NI. If this multiplicity is at most 2 , then the residue $A_{s 1}$ and the quadratic residue $A_{s 2}$ corresponding to $s_{0}$ are related to the residue $A_{1}$ and the quadratic residue $A_{2}$ corresponding to $z_{0}$ by: $A_{s 2}=\frac{1}{4} A_{2}$ and $A_{s 1}=\frac{1}{2}\left(A_{1}-A_{2}\right)$, since

$$
G(z)=G_{1}(z)+\frac{A_{1}}{z-1}+\frac{A_{2}}{(z-1)^{2}},
$$

where $G_{1}(z)$ is analytic in an open set containing $z_{0}=1$, and

$$
\begin{aligned}
G_{c}(s) & =G_{c, 1}(s)+\frac{A_{1}}{\frac{1+s}{1-s}-1}+\frac{A_{2}}{\left(\frac{1+s}{1-s}-1\right)^{2}} \\
& =\left(G_{c, 1}(s)-\frac{A_{1}}{2}+\frac{A_{2}}{4}\right)+\frac{A_{1}-A_{2}}{2 s}+\frac{A_{2}}{4 s^{2}} \\
& =\left(G_{c, 1}(s)-\frac{A_{1}}{2}+\frac{A_{2}}{4}\right)+\frac{A_{s 1}}{s}+\frac{A_{2 s}}{s^{2}},
\end{aligned}
$$

where $G_{c, 1}(s)-\frac{A_{1}}{2}+\frac{A_{2}}{4}$ is analytic in an open set containing $s_{0}=0$.

- $z_{0}=-1$ is a pole of $G(z)$ if and only if $\infty$ is a pole of $G_{c}$. Moreover, they are poles with the same multiplicity. If this multiplicity is strictly greater than 2 , then $G(z)$ is trivially not NI. In the case in which this multiplicity is at most 2, the first coefficient $A_{s 1}$ and the second coefficient $A_{s 2}$ in the expansion at infinity of $G_{c}(s)$ are connected to the residue $A_{1}$ and the quadratic residue $A_{2}$ corresponding to $z_{0}$ by: $A_{s 2}=\frac{1}{4} A_{2}$ and $A_{s 1}=-\frac{1}{2}\left(A_{1}+A_{2}\right)$ since

$$
G(z)=G_{1}(z)+\frac{A_{1}}{z+1}+\frac{A_{2}}{(z+1)^{2}},
$$

where $G_{1}(z)$ is analytic in an open set containing $z_{0}=-1$, 
and

$$
\begin{aligned}
G_{c}(s) & =G_{c, 1}(s)+\frac{A_{1}}{\frac{1+s}{1-s}+1}+\frac{A_{2}}{\left(\frac{1+s}{1-s}+1\right)^{2}} \\
& =\left(G_{c, 1}(s)+\frac{A_{1}}{2}+\frac{A_{2}}{4}\right)-\frac{A_{1}+A_{2}}{2} s+\frac{A_{2}}{4} s^{2} \\
& =\left(G_{c, 1}(s)+\frac{A_{1}}{2}+\frac{A_{2}}{4}\right)+A_{1 s} s+A_{2 s} s^{2},
\end{aligned}
$$

where $G_{c, 1}(s)+\frac{A_{1}}{2}+\frac{A_{2}}{4}$ is rational and proper.

Now, we apply [9, Lemma 3.1] in both directions and get the desired result.

Remark 3.2 In Definition 3.1 we need to assume symmetry of the transfer function matrix in order to introduce the notion of a NI system as a property that is defined in the domain of analyticity: this definition is the analogue to the classic definition of PR systems and has the important advantage of considering a general setting that does not require rationality assumptions. Note, however, that if one is only interested in the rational case, it is possible to consider conditions (i)-(v) of Lemma 3.2 as the definition of rational NI transfer functions and this clearly does not require any symmetry assumption. This is indeed the route taken in the first papers on continuous-time NI systems, see [12,20]. A similar observation can made for the definition of strictly negative imaginary systems given below.

The reader can check that, as long as one considers only rational transfer functions, all the results derived in this paper can be generalized to the case of non-symmetric transfer functions.

We now define the notions of strictly negative imaginary systems in discrete-time.

Definition 3.2 Let $G: \mathbb{C} \longrightarrow \mathbb{C}^{m \times m}$ be a discrete-time, real transfer function. Then, $G(z)$ is discrete-time strongly strictly negative imaginary (SSNI) if for some $\delta \in(0,1)$, the transfer function $G(\delta z)$ is NI and $i\left[G(z)-G(1 / z)^{\top}\right]$ has full normal rank.

Now, we show that SSNI as defined in Definition 3.2 can be equivalently checked via conditions on the domain of analyticity.

Lemma 3.3 Let $G: \mathbb{C} \longrightarrow \mathbb{C}^{m \times m}$ be a discrete-time, real transfer function. Then, $G(z)$ is SSNI if and only if there exists $\delta \in(0,1)$ such that

(i) $G(z)$ is analytic in $\{z \in \mathbb{C}:|z|>\delta\}$;

(ii) $i\left[G(z)-G(z)^{*}\right]>0$ for all $z \in \mathbb{C}$ such that $|z|>\delta$ and $\mathfrak{I m}(z)>0$

(iii) $i\left[G(z)-G(z)^{*}\right]=0$ for all $z \in \mathbb{C}$ such that $|z|>\delta$ and $\mathfrak{I m}(z)=0$; (iv) $i\left[G(z)-G(z)^{*}\right]<0$ for all $z \in \mathbb{C}$ such that $|z|>\delta$ and $\mathfrak{I m}(z)<0$.

Proof: Definition 3.2 trivially gives equivalence to the existence of $\delta \in(0,1)$ such that conditions (i)-(iv) are satisfied with non-strict inequalities in (ii) and (iv) on $i[G(z)-$ $\left.G(z)^{*}\right]$. We hence only need to show that the fact that $G$ is SSNI implies that the inequalities in (ii) and (iv) are indeed strict. We prove only that (ii) is strict since (iv) follows by symmetry. Let $G$ be analytic in $\mathbb{C}_{\delta} \stackrel{\text { def }}{=}\{z \in \mathbb{C}:|z|>\delta\}$ and assume by contradiction that there exist $z_{0} \in\{z \in \mathbb{C}$ : $|z|>\delta$ and $\mathfrak{I m}\{z\}>0\}$ and a nonzero vector $v$ such that $v^{*}\left(i\left[G\left(z_{0}\right)-G\left(z_{0}\right)^{*}\right]\right) v=0$. Since $G$ is analytic in $\mathbb{C}_{\delta}$, the function $h(z) \stackrel{\text { def }}{=} v^{*}\left(i\left[G(z)-G(z)^{*}\right]\right) v$ is harmonic in the same domain. Consider a real number $M>1$ such that $M>\left|z_{0}\right|$ and a real number $\delta_{1}$ such that $\delta<\delta_{1}<\left|z_{0}\right|$, so that $z_{0}$ is in the interior of the compact set $\mathscr{C} \stackrel{\text { def }}{=}\left\{z \in \mathbb{C}: \delta_{1} \leq|z| \leq\right.$ $M, \mathfrak{I m}\{z\} \geq 0\}$, which is contained in $\mathbb{C}_{\delta}$. Since $h(z)$ is non-negative in $\mathscr{C}$ and $h\left(z_{0}\right)=0$, then $h(z)$ restricted to $\mathscr{C}$ attains its minimum at a point $z_{0}$ in the interior of $\mathscr{C}$. Hence, $h(z)$ identically zero in $\mathscr{C}$ and hence, in particular, it is identically zero in the (upper half of the unit circle and, by symmetry, in the whole of the) unit circle. This is a contradiction, since $i\left[G(z)-G(1 / z)^{\top}\right]$ — that coincides with $h(z)$ in the unit circle - is required to have full normal rank by Definition 3.2.

We now specialize Lemma 3.3 to the unit disc. However, first we need a preliminary lemma.

Lemma 3.4 Let $g: \mathbb{C} \longrightarrow \mathbb{C}$ be a scalar discrete-time, real, rational, proper transfer function. Assume that $g(z)$ is a SSNI function. If $g(1)=0$ then the multiplicity of the zero in 1 of $g(z)$ is equal to 1 . Similarly, if $g(-1)=0$ then the multiplicity of the zero in -1 of $g(z)$ is equal to 1 .

Proof: Since $g(z)$ is a SSNI function, it has no poles in 1 and we can expand $g(z)$ at 1 as

$$
g(z)=\sum_{k=h}^{\infty} r_{k}(z-1)^{k}
$$

where $h$ is the multiplicity of the zero in 1 of $g(z), r_{h} \neq 0$, and $\limsup \sup _{k \rightarrow \infty}\left|r_{k}\right|^{1 / k}$ is finite so that there exists a constant $c$ such that $\left|r_{k}\right|^{1 / k}<c$ for all $k \geq h$. Let $z=1+\varepsilon e^{i \theta}$, with $\varepsilon>0$ and $0<\theta<\pi$. We have

$$
p(z) \stackrel{\text { def }}{=} i\left[g(z)-g(z)^{*}\right]=\varepsilon^{h}\left[-2 r_{h} \sin (h \theta)+\delta\right]
$$

with $\delta \stackrel{\text { def }}{=} \varepsilon^{-h} \sum_{k=h+1}^{\infty}-2 r_{k} \varepsilon^{k} \sin (k \theta)$. For $\varepsilon$ such that $|c \varepsilon|<$ 1 , we have

$$
|\delta| \leq \sum_{k=h+1}^{\infty}\left|2 r_{k} \varepsilon^{k-h} \sin (k \theta)\right| \leq \varepsilon \frac{2|c|^{h+1}}{1-c \varepsilon}
$$


which is arbitrarily small for a sufficiently small $\varepsilon$. We can then choose $\varepsilon$ such that $|\delta|<\left|2 r_{h}\right|$. Assume, by contradiction, that $h>1$ and define $\theta_{1} \stackrel{\text { def }}{=} \pi /(2 h)$ and $\theta_{2} \stackrel{\text { def }}{=} 3 \pi /(2 h)$ (notice that, if $h>1$, both $\theta_{1}$ and $\theta_{2}$ are in $(0, \pi)$ ). Then we have that $p\left(\varepsilon e^{i \theta_{1}}\right)=\varepsilon^{h}\left[-2 r_{h}+\delta\right]$ and $p\left(\varepsilon e^{i \theta_{2}}\right)=\varepsilon^{h}\left[2 r_{h}+\delta\right]$ have opposite signs which is a contradiction because $g(z)$ is a SSNI function so that $p\left(\varepsilon e^{i \theta}\right)$ is positive for any pair $\varepsilon>0$ and $0<\theta<\pi$. The proof for -1 is similar.

Theorem 3.1 Let $G: \mathbb{C} \longrightarrow \mathbb{C}^{m \times m}$ be a discrete-time, real, rational, proper transfer function. Then $G(z)$ is SSNI if and only if

(i) $G(z)$ has all its poles strictly inside the unit circle;

(ii) $i\left[G\left(e^{i \theta}\right)-G\left(e^{i \theta}\right)^{*}\right]>0$ for all $\theta \in(0, \pi)$;

(iii)

(iv)

$$
Q_{0} \stackrel{\text { def }}{=} \lim _{\theta \rightarrow 0^{+}} \frac{1}{\sin \theta} i\left[G\left(e^{i \theta}\right)-G\left(e^{i \theta}\right)^{*}\right]>0
$$

$$
Q_{\pi} \stackrel{\text { def }}{=} \lim _{\theta \rightarrow \pi^{-}} \frac{1}{\sin \theta} i\left[G\left(e^{i \theta}\right)-G\left(e^{i \theta}\right)^{*}\right]>0
$$

Proof: Necessity of (i) and (ii) is trivial from Lemma 3.3. We now prove necessity of (iii) (necessity of (iv) is similar). Assume that $G$ is SSNI. Then clearly the limit $Q_{0}$ defined in (iii) exists and is positive semi-definite. Assume by contradiction that $Q_{0}$ is singular and let $v \in \operatorname{ker} Q_{0}$. Let $g^{\prime}(z) \stackrel{\text { def }}{=} v^{\top} G(z) v$ and $g(z) \stackrel{\text { def }}{=} g^{\prime}(z)-g^{\prime}(1)$. Clearly, $g(z)$ is a rational proper SSNI function with a zero in 1 and such that

$$
\lim _{\theta \rightarrow 0^{+}} \frac{1}{\sin \theta} i\left[g\left(e^{i \theta}\right)-g\left(e^{i \theta}\right)^{*}\right]=0 .
$$

By expanding $g(z)$ around 1 as

$$
g(z)=\sum_{k=h}^{\infty} r_{k}(z-1)^{k}
$$

we see that (6) implies that $h>1$, which is a contradiction in view of Lemma 3.4 .

As for sufficiency, assume that $G(s)$ is real symmetric and rational and that it satisfies $(i)$, (ii), (iii) and (iv). We now show that we can choose $\rho<1$ in such a way that

$$
i\left[G\left(\rho e^{i \theta}\right)-G\left(\rho e^{i \theta}\right)^{*}\right]>0 \forall \theta \in(0, \pi) .
$$

In view of condition (ii), we have that for all $\pi>\theta_{2}>\theta_{1}>0$ there exists $\rho<1$ such that

$$
i\left[G\left(\rho e^{i \theta}\right)-G\left(\rho e^{i \theta}\right)^{*}\right]>0 \forall \theta \in\left[\theta_{1}, \theta_{2}\right],
$$

so that it is sufficient to show that given an arbitrarily small $\theta_{1}$ and an arbitrarily large $\theta_{2}$,

$$
i\left[G\left(\rho e^{i \theta}\right)-G\left(\rho e^{i \theta}\right)^{*}\right]>0 \forall \theta \in\left(0, \theta_{1}\right)
$$

and

$$
i\left[G\left(\rho e^{i \theta}\right)-G\left(\rho e^{i \theta}\right)^{*}\right]>0, \forall \theta \in\left(\theta_{2}, \pi\right) .
$$

As for (9), let $\delta \stackrel{\text { def }}{=} \rho e^{i \theta}-1$ with $\theta \in\left(0, \theta_{1}\right)$ and consider the following expansion of $G(\delta)$ :

$$
G(\delta)=D_{0}+\delta D_{1}+\delta^{2} D_{2}+\ldots
$$

which clearly converges for a sufficiently small $\delta$ (if we considered a minimal realization $G(z)=C(z I-A)^{-1} B+D$, we would have $D_{0} \stackrel{\text { def }}{=} D-C(I-A)^{-1} B$ and $D_{i} \stackrel{\text { def }}{=}-C(I-$ $A)^{-i-1} B$ for $\left.i>1\right)$. Since $G(z)$ is real symmetric by our standing assumption, we have $D_{i}=D_{i}^{\top}$. Moreover, $Q_{0} \stackrel{\text { def }}{=}$ $\lim _{\theta \rightarrow 0^{+}}(1 / \sin \theta) i\left[G\left(e^{i \theta}\right)-G\left(e^{i \theta}\right)^{*}\right]=-2 D_{1}$, so that by (iv), we have $D_{1}<0$. A direct calculation gives

$$
\begin{aligned}
i\left[G\left(\rho e^{i \theta}\right)-G\left(\rho e^{i \theta}\right)^{*}\right]= & -\rho \sin (\theta) 2 D_{1} \\
& +i \sum_{j=2}^{\infty}\left[\delta^{j}-\left(\delta^{*}\right)^{j}\right] D_{j}
\end{aligned}
$$

Now we observe that

$$
i \sum_{j=2}^{\infty}\left[\delta^{j}-\left(\delta^{*}\right)^{j}\right] D_{j}=-2 \rho \sin \theta \sum_{j=2}^{\infty} \sum_{k=0}^{j-1}\left[\delta^{k}\left(\delta^{*}\right)^{j-1-k}\right] D_{j}
$$

so that

$$
\begin{aligned}
\left\|i \sum_{j=3}^{\infty}\left[\delta^{j}-\left(\delta^{*}\right)^{j}\right] D_{j}\right\| & \leq 2 \rho \sin \theta \sum_{j=2}^{\infty} j|\delta|^{j-1}\left\|D_{j}\right\| \\
& =2 \rho \sin \theta|\delta| \sum_{j=2}^{\infty} j|\delta|^{j-2}\left\|D_{j}\right\| \\
& \leq 2 \rho \sin \theta|\delta| \sigma
\end{aligned}
$$

for a certain $\sigma$ which remains bounded as $|\delta|$ tends to zero. Since, by choosing a sufficiently small $\delta$ we can make $-D_{1}>\sigma|\delta| I$, we have (9). The proof of (10) is similar.

In analogy with the continuous-time case [8], we introduce the following definition of a weaker notion of strictly negative imaginary systems.

Definition 3.3 The discrete-time, real, rational, proper transfer function $G: \mathbb{C} \longrightarrow \mathbb{C}^{m \times m}$ is discrete-time weakly strictly negative imaginary (WSNI) if it satisfies conditions (i) and (ii) of Theorem 3.1.

The next lemma shows that the definition of WSNI characterizes properties on the outside of the unit disk too.

Lemma 3.5 Let $G: \mathbb{C} \longrightarrow \mathbb{C}^{m \times m}$ be a discrete-time, real, rational, proper transfer function. Then, $G(z)$ is WSNI if and only if there exists $\delta \in(0,1)$ such that

(i) $G(z)$ is analytic in $\{z \in \mathbb{C}:|z|>\delta\}$; 
(ii) $i\left[G(z)-G(z)^{*}\right]>0$ for all $z \in \mathbb{C}$ such that $|z| \geq 1$ and $\mathfrak{I m}(z)>0$

(iii) $i\left[G(z)-G(z)^{*}\right]=0$ for all $z \in \mathbb{C}$ such that $|z| \geq 1$ and $\mathfrak{I m}(z)=0$

(iv) $i\left[G(z)-G(z)^{*}\right]<0$ for all $z \in \mathbb{C}$ such that $|z| \geq 1$ and $\mathfrak{I m}(z)<0$.

Proof: Sufficiency is trivial by restricting on $\{z \in \mathbb{C}:|z|=$ $1\}$. Necessity can be proven as follows: if $G$ is WSNI, then (i) is satisfied and $G$ is NI (from Lemma 3.2). If $G$ is NI, then (ii)-(iv) in Definition 3.1 are satisfied. Strict inequalities in conditions (ii) and (iv) outside the unit circle are then obtained via an argument similar to that given in the proof of Lemma 3.3. Appending the $\{z \in \mathbb{C}:|z|=1\}$ properties of $G$ to the conditions (ii)-(iv) in Definition 3.1 (since $G$ is WSNI) yields (ii)-(iv) above since $G$ fulfils $(i)$ above.

The following lemma relates the strong classes with the weak classes with the non-strict classes of negative imaginary systems.

Lemma 3.6 The set of SSNI systems is contained in the set of WSNI systems which is in turn contained in the set of NI systems.

Proof: Trivial from the definitions.

The following lemma relates a NI system with a PR system.

Lemma 3.7 Let $G: \mathbb{C} \longrightarrow \mathbb{C}^{m \times m}$ be a discrete-time, symmetric, real, rational, proper transfer function with no poles at $z=-1$. Then, $G(z)$ is NI if and only if

$F(z)=\frac{z-1}{z+1}[G(z)-G(-1)]$

is $P R$.

Proof: (Only if). The set of poles of $F(z)$ is contained in the set of poles of $G(z)$ (in fact, in (11) the pole in -1 of $\frac{z-1}{z+1}$ is cancelled by the zero in -1 of $[G(z)-G(-1)])$. Since $G(z)$ is a symmetric, real, rational, proper, NI transfer function, $F(z)$ is analytic in $|z|>1$. Let $\theta_{0} \in(0, \pi)$, and assume that $z=e^{i \theta_{0}}$ is not a pole of $G(z)$. Then, $z=e^{i \theta_{0}}$ is not a pole of $F(z)$, and a simple calculation gives

$F\left(e^{i \theta_{0}}\right)+F\left(e^{i \theta_{0}}\right)^{*}=\frac{\sin \theta_{0}}{1+\cos \theta_{0}} i\left[G\left(e^{i \theta_{0}}\right)-G\left(e^{i \theta_{0}}\right)^{*}\right] \geq 0$

in view of Lemma 3.2.

Let us now assume that $z=e^{i \theta_{0}}$, with $\theta_{0} \in(0, \pi)$, is a pole of $G(z)$. From Lemma 3.2, it is a simple pole, and from (11) it is also a simple pole of $F(z)$. We can write

$$
G(z)=G_{1}(z)+\frac{A}{z-e^{i \theta_{0}}},
$$

where $G_{1}(z)$ is a rational function which is analytic in an open set containing $z=e^{i \theta_{0}}$ and the matrix $A$ is non-zero. Then,

$$
\begin{aligned}
K_{0} & =e^{-i \theta_{0}} \lim _{z \rightarrow e^{i \theta_{0}}}\left(z-e^{i \theta_{0}}\right) i G(z) \\
& =e^{-i \theta_{0}} \lim _{z \rightarrow e^{i \theta_{0}}}\left(z-e^{i \theta_{0}}\right) i\left(G_{1}(z)+\frac{A}{z-e^{i \theta_{0}}}\right)=i e^{-i \theta_{0}} A
\end{aligned}
$$

is Hermitian and positive semidefinite. The normalized residue of $F(z)$ in $e^{i \theta_{0}}$ is given by

$$
\begin{aligned}
e^{-i \theta_{0}} & \lim _{z \rightarrow e^{i \theta_{0}}}\left(z-e^{i \theta_{0}}\right) F(z) \\
= & e^{-i \theta_{0}} \lim _{z \rightarrow e^{i \theta_{0}}} \frac{z-1}{z+1}\left[\left(z-e^{i \theta_{0}}\right) G(z)-\left(z-e^{i \theta_{0}}\right) G(-1)\right] \\
& =e^{-i \theta_{0}} \frac{e^{i \theta_{0}}-1}{e^{i \theta_{0}}+1} A=\frac{\sin \theta_{0}}{1+\cos \theta_{0}} i e^{-i \theta_{0}} A \geq 0 .
\end{aligned}
$$

Let us now consider the case $\theta_{0}=0$, i.e., $z_{0}=e^{i \theta_{0}}=1$. If $G(z)$ has no poles at $z_{0}=1$, neither does $F(z)$. In this case, $F(1)=0$, which gives $F(1)+F(1)^{*}=0 \geq 0$. If $G(z)$ has a simple pole at $z_{0}=1$, then $F(z)$ has no poles at $z_{0}=1$. In this case, $G(z)=G_{1}(z)+\frac{A}{z-1}$, where $G_{1}(z)$ is a rational function which is analytic in an open set containing $z_{0}=1$, and where $A \geq 0$ from (iv) in Lemma 3.2 (because the quadratic residual is zero). Thus,

$$
F(z)=\frac{z-1}{z+1}\left[G_{1}(z)+\frac{A}{z-1}-G(-1)\right]
$$

so that $F(1)=A / 2$, and $F(1)+F(1)^{*}=A \geq 0$. Now, consider the case in which $G(z)$ has a double pole at $z_{0}=1$. In this case, we can write $G(z)=G_{1}(z)+\frac{A_{1}}{z-1}+\frac{A_{2}}{(z-1)^{2}}$, where $G_{1}(z)$ is a rational function which is analytic in an open set containing $z_{0}=1, A_{1} \geq A_{2}$ and $A_{2} \geq 0$. In this case,

$$
\begin{aligned}
F(z)= & \frac{z-1}{z+1}\left[G_{1}(z)+\frac{A_{1}}{z-1}+\frac{A_{2}}{(z-1)^{2}}-G(-1)\right] \\
= & {\left[\frac{z-1}{z+1} G_{1}(z)+\frac{A_{1}}{z+1}-\frac{z-1}{z+1} G(-1)-\frac{A_{2}}{2(z+1)}\right] } \\
& +\frac{A_{2}}{2(z-1)} .
\end{aligned}
$$

Since $G_{1}(z)$ is analytic in an open set containing $z_{0}=1$, $\frac{z-1}{z+1} G_{1}(z)+\frac{A_{1}}{z+1}-\frac{z-1}{z+1} G(-1)-\frac{A_{2}}{2(z+1)}$ is also analytic in an open set containing $z_{0}=1$. Thus, $F(z)$ has a simple pole at $z_{0}=1$, and the corresponding residue $A_{2} / 2$ is positive semidefinite (notice that in this case the residue and the normalized residue coincide because $z_{0}=1$ ).

Let us finally consider the case $\theta_{0}=\pi$, i.e., $z_{0}=e^{i \theta_{0}}=-1$. We know that $G(-1)$ is finite and hence $F(-1)$ is finite as well. Moreover, $F\left(e^{i \theta_{0}}\right)+F\left(e^{i \theta_{0}}\right)^{*}$ is positive semidefinite for all $\theta_{0} \in(0, \pi)$ that is not a pole of $G(z)$. Therefore, by continuity, we have $F(-1)+F(-1)^{*} \geq 0$. 
(If). Let $F$ be given by (11). Since $F(z)$ is symmetric, real, rational, proper, discrete-time positive real and $G(-1)=$ $G(-1)^{\top}$, it is sufficient to show that

$$
G_{0}(z) \stackrel{\text { def }}{=} \frac{z+1}{z-1} F(z)
$$

is NI because $G_{0}(z)$ is NI if and only if $G(z)=G_{0}(z)+$ $G(-1)$ is NI. We observe that $G_{0}(z)$ is proper, symmetric, real, rational, discrete-time and analytic in $|z|>1$. Also, $F(z)$ and $G_{0}(z)$ have the same poles, with the possible exception of a pole at $z=1$. Notice that $F(z)$ does not have a pole at $z=-1$ due to its construction in (11). Let $z_{0}=e^{i \theta_{0}}$ with $\theta_{0} \in(0, \pi)$. Assume $z_{0}$ is not a pole of $F(z)$. Then, it is not a pole of $G_{0}(z)$. We find

$G_{0}\left(e^{i \theta_{0}}\right)=\frac{e^{i \theta_{0}}+1}{e^{i \theta_{0}}-1} F\left(e^{i \theta_{0}}\right)=-\frac{i \sin \theta_{0}}{1-\cos \theta_{0}} F\left(e^{i \theta_{0}}\right)$,

so that

$i\left[G_{0}\left(e^{i \theta_{0}}\right)-G_{0}\left(e^{i \theta_{0}}\right)^{*}\right]=\frac{\sin \theta_{0}}{1-\cos \theta_{0}}\left[F\left(e^{i \theta_{0}}\right)+F\left(e^{i \theta_{0}}\right)^{*}\right] \geq 0$,

because $F\left(e^{i \theta_{0}}\right)+F\left(e^{i \theta_{0}}\right)^{*} \geq 0$. We now assume that $z_{0}=$ $e^{i \theta_{0}}$ with $\theta_{0} \in(0, \pi)$ is a pole of $F(z)$. Then, it is also a pole of $G_{0}(z)$. Since $F(z)$ is PR, $z_{0}$ is a simple pole. Thus, $z_{0}$ is also a simple pole of $G_{0}(z)$. Moreover, the matrix $K_{0}=e^{-i \theta_{0}} \lim _{z \rightarrow e^{i \theta_{0}}}\left(z-e^{i \theta_{0}}\right) F(z)$ is positive semidefinite, see Theorem 2.1 . This then implies that

$$
\begin{aligned}
e^{-i \theta_{0}} \lim _{z \rightarrow z_{0}}\left(z-e^{i \theta_{0}}\right) i G_{0}(z) & =e^{-i \theta_{0}} \lim _{z \rightarrow z_{0}} i \frac{z+1}{z-1}\left(z-e^{i \theta_{0}}\right) F(z) \\
& =i \frac{e^{i \theta_{0}}+1}{e^{i \theta_{0}}-1} K_{0} \\
& =\frac{\sin \theta_{0}}{1-\cos \theta_{0}} K_{0} \geq 0
\end{aligned}
$$

When $z=1, F(z)$ can either have no poles or a simple pole. Assume $z=1$ is not a pole. Then, $G_{0}(z)=G_{1}(z)+$ $\frac{2 F(1)}{z-1}$ where $G_{1}(z)$ is analytic in a region near $z=1$. Then, $K_{0}=\lim _{z \rightarrow 1}(z-1) G_{0}(z)=2 F(1)=F(1)+F(1)^{\top}$ (due to $F(z)$ being symmetric), which is non-negative in view of Theorem 2.1.

Assume now that $z=1$ is a simple pole of $F(z)$. We can write $F(z)=F_{1}(z)+\frac{A}{z-1}$, where $F_{1}(z)$ is analytic near $z=1$ and $0 \leq A \leq 2 F_{1}$ (1) (via Theorem 2.1, since $A \geq 0$ directly from the theorem statement and $0 \leq F\left(e^{i \theta}\right)+F\left(e^{i \theta}\right)^{*}=F_{1}\left(e^{i \theta}\right)+$ $F_{1}\left(e^{i \theta}\right)^{*}-A$ implies $A \leq 2 F_{1}(1)$ in the limit as $\theta \rightarrow 0$ due to continuity and $F_{1}(1)$ being symmetric).

Hence,

$$
\begin{aligned}
G_{0}(z) & =\frac{z+1}{z-1} F(z)=\frac{z+1}{z-1} F_{1}(z)+\frac{z+1}{(z-1)^{2}} A \\
& =G_{2}(z)+\frac{2 F_{1}(1)+A}{z-1}+\frac{2 A}{(z-1)^{2}}
\end{aligned}
$$

where $G_{2}(z)$ is analytic in the neighbourhood of $z=1$. Thus, the residue and the quadratic residue are $A_{1}=A+2 F_{1}(1)$ and $A_{2}=2 A$, and the condition that ensures that $F(z)$ is PR now guarantees that $A_{2} \geq 0$ and $A_{1} \geq A_{2}$, so that $G_{0}(z)$ is NI.

Lemma 3.8 Let $G: \mathbb{C} \longrightarrow \mathbb{C}^{m \times m}$ be a discrete-time, real, rational, proper, NI transfer function with no poles at $z=-1$. Then

- $G(\infty)=G(\infty)^{\top}$;

- $G(-1)$ exists and $G(-1)=G(-1)^{\top}$.

Furthermore, let $\left[\begin{array}{l|l}A & B \\ \hline C & D\end{array}\right]$ be a minimal state-space realization of $G(z)$. Then,

- $C(I+A)^{-1} B=B^{\top}\left(I+A^{\top}\right)^{-1} C^{\top}$;

- $F(z)=\frac{z-1}{z+1}[G(z)-G(-1)]$ has a state-space realization

$$
\left[\begin{array}{c|c}
A & B \\
\hline C(A-I)(A+I)^{-1} & C(A+I)^{-1} B
\end{array}\right]
$$

which is minimal when A has no eigenvalues at 1.

Proof: Since $G(z)$ is symmetric, i.e. $G(z)=G(z)^{\top}$ for all $|z|>1$, we obtain $G(\infty)=G(\infty)^{\top}$ via a limiting argument. Since $G(z)$ has no poles at $z=-1$, it follows that $G(-1)$ exists. Now, $G(z)=G(z)^{\top}$ for all $|z|>1$ implies that $G(-1)=G(-1)^{\top}$ via continuity and a limiting argument.

From $G(-1)=G(-1)^{\top}$ and $D=D^{\top}$, it immediately follows that $C(I+A)^{-1} B=B^{\top}\left(I+A^{\top}\right)^{-1} C^{\top}$.

Let us now consider a state-space realization of $F(z)$. A realization of the transfer function matrix $\frac{z-1}{z+1} I$ is given by $\left[\begin{array}{c|c}-I & I \\ \hline-2 I & I\end{array}\right]$, while a realization of the term $G(z)-G(-1)=$ $C(z I-A)^{-1}+C(A+I)^{-1} B$ is given by $\left[\begin{array}{c|c}A & B \\ \hline C & C(A+I)^{-1} B\end{array}\right]$. Thus, a realization for $F(z)$ is given by

$$
\left[\begin{array}{cc|c}
-I & C & C(A+I)^{-1} B \\
0 & A & B \\
\hline-2 I & C & C(A+I)^{-1} B
\end{array}\right]
$$


Changing state coordinates via

$$
T=\left[\begin{array}{cc}
I & C(I+A)^{-1} \\
0 & I
\end{array}\right]
$$

yields

$$
\begin{aligned}
F(z) & =\left[\begin{array}{ll|l}
-I & 0 & 0 \\
0 & A & B \\
\hline-2 I C\left[I-2(I+A)^{-1}\right] & C(A+I)^{-1} B
\end{array}\right] \\
& =\left[\begin{array}{cc|c}
-I & 0 & 0 \\
0 & A & B \\
\hline-2 I C(A-I)(I+A)^{-1} & C(A+I)^{-1} B
\end{array}\right] .
\end{aligned}
$$

This realization is not minimal because it is easily seen that it is not completely reachable. Eliminating the non-reachable part one obtains

$F(z)=\left[\begin{array}{c|c}A & B \\ \hline C(A-I)(A+I)^{-1} & C(A+I)^{-1} B\end{array}\right]$,

which is minimal if $\operatorname{det}(A-I) \neq 0$.

Remark 3.3 We have derived the condition $G(-1)=$ $G(-1)^{\top}$ as a consequence of the symmetry of $G(z)$. However, if we consider, in the spirit of Remark 3.2, the possibly non-symmetric case, then condition $G(-1)=G(-1)^{\top}$ still holds. More precisely, assuming that rational NI systems are defined by conditions (i)-(v) of Lemma 3.2 (and that symmetry is not assumed), we have that if -1 is not a pole of $G(z)$ then $G(-1)=G(-1)^{\top}$. In fact, since by condition (ii) of Lemma 3.2, $i\left[G\left(e^{i \theta}\right)-G\left(e^{i \theta}\right)^{*}\right] \geq 0$ for all $\theta \in(0, \pi)$ (except for the values of $\theta$ for which $z=e^{i \theta}$ is a pole of $G(z)$ ), we can use continuity and conclude that $i\left[G(-1)-G(-1)^{*}\right] \geq 0$, but $G(-1)$ is real so that $i\left[G(-1)-G(-1)^{\top}\right]$ is positive semi-definite. The diagonal entries of $i\left[G(-1)-G(-1)^{\top}\right]$ are zero so that we necessarily have $G(-1)-G(-1)^{\top}=0$. Similarly, assuming that rational NI systems are defined by conditions (i)-(v) of Lemma 3.2 (and that symmetry is not assumed), we have that if 1 is not a pole of $G(z)$ then $G(1)=G(1)^{\top}$.

In this non-symmetric setting, it is easy to check that the result analogue to Lemma 3.7 is that $G(z)$ without poles in -1 is NI if and only if $F(z)$ defined by (11) is PR and $G(-1)=G(-1)^{\top}$.

We are now in a position to give a discrete-time negative imaginary lemma that gives a complete state-space characterization of NI systems. Different grades of strength of continuous-time negative imaginary lemmas are given in $[12,13,23]$
Theorem 3.2 Let $\left[\begin{array}{l|l}A & B \\ \hline C & D\end{array}\right]$ be a minimal state-space realization of a discrete-time, symmetric, real, rational, proper transfer function $G(z)$. Suppose $\operatorname{det}(I+A) \neq 0$ and $\operatorname{det}(I-$ $A) \neq 0$. Then, $G(z)$ is NI if and only if there exists a real matrix $X=X^{\top}>0$ such that

$$
X-A^{\top} X A \geq 0 \quad \text { and } \quad C=-B^{\top}\left(A^{\top}-I\right)^{-1} X(A+I) .
$$

Proof: First, note that

$A(A-I)^{-1}=I+(A-I)^{-1}$.

Now, in view of Lemma 3.7, $G(z)$ is NI if and only if $F(z)=$ $\frac{z-1}{z+1}[G(z)-G(-1)]$ is PR. By Lemma 3.8, this is equivalent
to

$$
\left[\begin{array}{c|c}
A & B \\
\hline C(A-I)(A+I)^{-1} & C(A+I)^{-1} B
\end{array}\right]
$$

being PR. Using Lemma 2.1, the latter conditions are equivalent to existence of $X>0$ and $L, W$ such that

$$
\begin{aligned}
& X-A^{\top} X A=L^{\top} L, \\
& \left(A^{\top}+I\right)^{-1}\left(A^{\top}-I\right) C^{\top}-A^{\top} X B=L^{\top} W, \\
& C(A+I)^{-1} B+B^{\top}\left(A^{\top}+I\right)^{-1} C^{\top}-B^{\top} X B=W^{\top} W .
\end{aligned}
$$

Eq. (15) can be written as

$$
C=\left(W^{\top} L+B^{\top} X A\right)(A-I)^{-1}(A+I),
$$

which can be substituted into (16) to give

$$
\begin{array}{r}
B^{\top} X\left[I+(A-I)^{-1}\right] B+B^{\top}\left[I+\left(A^{\top}-I\right)^{-1}\right] X B-B^{\top} X B \\
=W^{\top} W-W^{\top} L(A-I)^{-1} B-B^{\top}\left(A^{\top}-I\right)^{-1} L^{\top} W
\end{array}
$$

in view of (13). This equation can also be written as

$$
\begin{aligned}
& B^{\top} X(A-I)^{-1} B+B^{\top}\left(A^{\top}-I\right)^{-1} X B+B^{\top} X B \\
& +B^{\top}\left(A^{\top}-I\right)^{-1} L^{\top} L(A-I)^{-1} B \\
& \quad=\left[W-L(A-I)^{-1} B\right]^{\top}\left[W-L(A-I)^{-1} B\right] .
\end{aligned}
$$

Substituting the term $L^{\top} L$ of (14) into the latter yields

$$
\begin{aligned}
& B^{\top} X(A-I)^{-1} B+B^{\top}\left(A^{\top}-I\right)^{-1} X B+B^{\top} X B+ \\
& B^{\top}\left(A^{\top}-I\right)^{-1} X(A-I)^{-1} B-B^{\top}\left(A^{\top}-I\right)^{-1} A^{\top} X A(A-I)^{-1} B \\
& \quad=\left[W-L(A-I)^{-1} B\right]^{\top}\left[W-L(A-I)^{-1} B\right] .
\end{aligned}
$$

Using (13), it is easily seen that the left hand-side of this equation is equal to zero, so that $W=L(A-I)^{-1} B$. This means that $G(z)$ is NI if and only if there exists $X>0$ such that $X-A^{\top} X A \geq 0$ and $C=\left[B^{\top}\left(A^{\top}-I\right)^{-1}\left(X-A^{\top} X A\right)+\right.$ $\left.B^{\top} X A\right](A-I)^{-1}(A+I)$. 
Now, using (13), $G(z)$ is NI if and only if there exists $X>$ 0 such that $X-A^{\top} X A \geq 0$ and $C(A+I)^{-1}=-B^{\top}\left(A^{\top}-\right.$ I) $)^{-1} X$.

The conditions given in Theorem 3.2 and Corollary 3.1 are non-strict linear matrix inequalities in the Lyapunov variable $X$ or $Y$ which yield a set of convex conditions that can be solved via commercially available software. The conditions are necessary and sufficient. If one were to tighten the nonstrict inequality to a strict inequality, a subset of negative imaginary systems would be obtained, see [13] for detailed discussions on this in the continuous-time setting.

Corollary 3.1 Let the suppositions of Theorem 3.2 hold. Then $G(z)$ is NI if and only if there exists $Y=Y^{\top}>0$ such that $Y-A Y A^{\top} \geq 0$ and $B=-(A-I) Y\left(A^{\top}+I\right)^{-1} C^{\top}$.

Proof: The result follows by letting $Y=X^{-1}$ and noting that $X-A^{\top} X A \geq 0$ is equivalent to $\left[\begin{array}{ll}X & A^{\top} \\ A & X^{-1}\end{array}\right] \geq 0$, which is in turn equivalent to $X^{-1}-A X^{-1} A^{\top} \geq 0$.

We next show that $G(1)$ and $G(-1)$ can be ordered for discrete-time negative imaginary systems.

Lemma 3.9 Let $G: \mathbb{C} \longrightarrow \mathbb{C}^{m \times m}$ be a discrete-time, real, rational, proper, NI (resp. WSNI) transfer function with no poles at +1 and -1 . Then

$$
G(1)-G(-1) \geq 0(\text { resp. }>0) .
$$

Proof: Using Theorem 3.2 and a minimal realization for $G(z)$, we find

$$
\begin{aligned}
G(1)-G(-1) & =C(I-A)^{-1} B+D-C(-I-A)^{-1} B-D \\
& =C\left[(I-A)^{-1}+(I+A)^{-1}\right] B \\
& =2 C(I+A)^{-1}(I-A)^{-1} B \\
& =-2 B^{\top}\left(A^{\top}-I\right)^{-1} X(I-A)^{-1} B \\
& =2 B^{\top}(I-A)^{-\top} X(I-A)^{-1} B \geq 0 .
\end{aligned}
$$

This concludes the proof when $G$ is NI.

Now, we focus on $G$ being WSNI. The strict inequality result will be proven via a contra-positive argument. Suppose there exists an $x \in \mathbb{R}^{m}$ such that $[G(1)-G(-1)] x=0$. Then $B^{\top}(I-A)^{-\top} X(I-A)^{-1} B x=0$ which implies that $B x=0$ as $X>0$. This then implies that $G\left(e^{i \theta}\right) x=D x \forall \theta \in(0, \pi)$, i.e.,

$$
\left(G\left(e^{i \theta}\right)-D\right) x=0, \quad \forall \theta \in(0, \pi) .
$$

Since $G$ is WSNI, $i\left[G\left(e^{i \theta}\right)-G\left(e^{i \theta}\right)^{*}\right]$ is positive definite for all $\theta \in(0, \pi)$ so that if, for $\theta_{0} \in(0, \pi), x$ is such that $x^{*}\left[i\left(G\left(e^{i \theta_{0}}\right)-G\left(e^{i \theta_{0}}\right)^{*}\right)\right] x=0$, we can conclude that $x=0$. Now recall that $D=D^{\top}$. Hence, $x^{*}\left[i\left(G\left(e^{i \theta_{0}}\right)-\right.\right.$ $\left.\left.G\left(e^{i \theta_{0}}\right)^{*}\right)\right] x=i x^{*}\left[\left(G\left(e^{i \theta_{0}}\right)-D\right)-\left(G\left(e^{i \theta_{0}}\right)-D\right)^{*}\right] x=0$. Hence $x=0$, so that $[G(1)-G(-1)]$ must be nonsingular. This completes the proof.

\section{Feedback interconnections and internal stability}

The following result shows under what circumstances are NI, WSNI and SSNI properties preserved when such systems are interconnected in feedback. Given complex matrices $S_{1}, S_{2}$ and complex vectors $y_{1}, y_{2}, u_{1}, u_{2}, \alpha, \beta$ of compatible dimension satisfying $\left[\begin{array}{l}y_{1} \\ \alpha\end{array}\right]=S_{1}\left[\begin{array}{c}u_{1} \\ \beta\end{array}\right]$ and $\left[\begin{array}{l}\beta \\ y_{2}\end{array}\right]=S_{2}\left[\begin{array}{l}\alpha \\ u_{2}\end{array}\right]$, let $S_{1} \star S_{2}$ denote the Redheffer star product which maps $\left[\begin{array}{l}u_{1} \\ u_{2}\end{array}\right]$ to $\left[\begin{array}{l}y_{1} \\ y_{2}\end{array}\right]$. Furthermore, let $F_{\ell}\left(S_{1}, S_{2}^{(1,1)}\right)\left(\operatorname{resp} . F_{u}\left(S_{2}, S_{1}^{(2,2)}\right)\right)$ denote the lower (resp. upper) linear fractional transformation. Finally, let $[P, Q]$ denote the positive feedback interconnection between systems $P$ and $Q$.

Lemma 4.1 Let $S_{1}: \mathbb{C} \rightarrow \mathbb{C}^{m_{1} \times m_{1}}$ be $N I$ (resp. WSNI or SSNI) and $S_{2}: \mathbb{C} \rightarrow \mathbb{C}^{m_{2} \times m_{2}}$ be NI (resp. WSNI or SSNI). Let $0<a, b \leq \min \left\{m_{1}, m_{2}\right\}$ and suppose the feedback interconnection corresponding to the Redheffer Star product $S_{1} \star S_{2}$ be internally stable. ${ }^{2}$ Then $S_{1} \star S_{2}$ is NI (resp. WSNI or SSNI).

Furthermore, if

- $a=b=m_{2}<m_{1}$, then $S_{1} \star S_{2}=F_{\ell}\left(S_{1}, S_{2}\right)$;

- $a=b=m_{1}<m_{2}$, then $S_{1} \star S_{2}=F_{u}\left(S_{2}, S_{1}\right)$;

- $a=b=m_{2}=m_{1} / 2, S_{1}=\left[\begin{array}{cc}P & I_{a} \\ I_{a} & 0\end{array}\right]$ and $S_{2}=Q$, then $S_{1} \star$ $S_{2}=P+Q$

- $a=b=m_{2}=m_{1} / 2, S_{1}=\left[\begin{array}{cc}0 & I_{a} \\ I_{a} & P\end{array}\right]$ and $S_{2}=Q$, then $S_{1}$ * $S_{2}=Q\left(I_{a}-P Q\right)^{-1}$

- $2 a=2 b=m_{1}=m_{2}, S_{1}=\left[\begin{array}{cc}0 & I_{a} \\ I_{a} & P\end{array}\right]$ and $S_{2}=\left[\begin{array}{cc}Q & I_{a} \\ I_{a} & 0\end{array}\right]$, then $S_{1} \star S_{2}=\left[\begin{array}{cc}-P & I_{a} \\ I_{a} & -Q\end{array}\right]^{-1}=\left[\begin{array}{cc}Q\left(I_{a}-P Q\right)^{-1} & \left(I_{a}-Q P\right)^{-1} \\ \left(I_{a}-P Q\right)^{-1} & P\left(I_{a}-Q P\right)^{-1}\end{array}\right]$ which corresponds to the positive feedback interconnection $[P, Q]$.

\footnotetext{
2 This is the standard meaning of "internal stability", i.e. add two extra exogenous input signals to the internal signals and ensure that all output signals and all internal signals are energy-bounded for any energy-bounded exogenous input excitation.
} 
Proof: Given $S_{1}(z), S_{2}(z)$ and complex vectors $y_{1}, y_{2}, u_{1}, u_{2}, \alpha, \beta$ of compatible dimension satisfying $\left[\begin{array}{l}y_{1} \\ \alpha\end{array}\right]=S_{1}(z)\left[\begin{array}{l}u_{1} \\ \beta\end{array}\right]$ and $\left[\begin{array}{l}\beta \\ y_{2}\end{array}\right]=S_{2}(z)\left[\begin{array}{l}\alpha \\ u_{2}\end{array}\right]$, it follows that $\left[\begin{array}{l}y_{1} \\ y_{2}\end{array}\right]=S_{1}(z) \star S_{2}(z)\left[\begin{array}{l}u_{1} \\ u_{2}\end{array}\right]$. Then, for all $\left[\begin{array}{l}u_{1} \\ \beta\end{array}\right] \in \mathbb{C}^{m_{1}},\left[\begin{array}{c}\alpha \\ u_{2}\end{array}\right] \in \mathbb{C}^{m_{2}}$ :

$$
\begin{aligned}
& {\left[\begin{array}{ll}
u_{1}^{*} & u_{2}^{*}
\end{array}\right]\left[i\left(\left[S_{1}(z) \star S_{2}(z)\right]-\left[S_{1}(z) \star S_{2}(z)\right]^{*}\right)\right]\left[\begin{array}{l}
u_{1} \\
u_{2}
\end{array}\right]} \\
& =i\left[\begin{array}{ll}
u_{1}^{*} & u_{2}^{*}
\end{array}\right]\left[\begin{array}{l}
y_{1} \\
y_{2}
\end{array}\right]-i\left[\begin{array}{ll}
y_{1}^{*} & y_{2}^{*}
\end{array}\right]\left[\begin{array}{l}
u_{1} \\
u_{2}
\end{array}\right] \\
& =i\left[\begin{array}{ll}
u_{1}^{*} & \beta^{*}
\end{array}\right]\left[\begin{array}{c}
y_{1} \\
\alpha
\end{array}\right]-i\left[\begin{array}{ll}
y_{1}^{*} & \alpha^{*}
\end{array}\right]\left[\begin{array}{c}
u_{1} \\
\beta
\end{array}\right] \\
& +i\left[\begin{array}{ll}
\alpha^{*} & u_{2}^{*}
\end{array}\right]\left[\begin{array}{c}
\beta \\
y_{2}
\end{array}\right]-i\left[\begin{array}{ll}
\beta^{*} & y_{2}^{*}
\end{array}\right]\left[\begin{array}{c}
\alpha \\
u_{2}
\end{array}\right] \\
& =\left[\begin{array}{ll}
u_{1}^{*} & \beta^{*}
\end{array}\right]\left[i\left(S_{1}(z)-S_{1}(z)^{*}\right)\right]\left[\begin{array}{c}
u_{1} \\
\beta
\end{array}\right] \\
& +\left[\begin{array}{ll}
\alpha^{*} & u_{2}^{*}
\end{array}\right]\left[i\left(S_{2}(z)-S_{2}(z)^{*}\right)\right]\left[\begin{array}{c}
\alpha \\
u_{2}
\end{array}\right] .
\end{aligned}
$$

Since the Redheffer star interconnection is internally stable, the three respective results (NI, WSNI, SSNI) then follow by applying Definition 3.1, Lemma 3.3 or Lemma 3.5 respectively on the corresponding domains of $z \in \mathbb{C}$ for $S_{1}(z)$ and $S_{2}(z)$.

The five cases where $a, b, S_{1}$ and $S_{2}$ are restricted are trivial consequences of a Redheffer calculation.

Example 4.1 This example shows that it is not possible to mix and match properties of $S_{1}$ and $S_{2}$ for the strict results in Lemma 4.1 to hold.

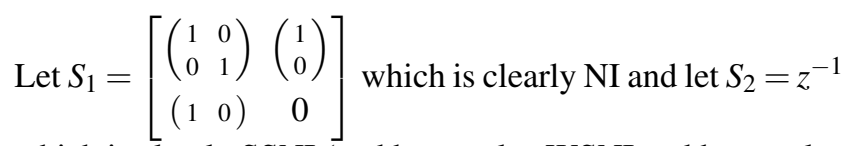
which is clearly SSNI (and hence also WSNI and hence also $\mathrm{NI}$ ). Then $S_{1} \star S_{2}=\left[\begin{array}{cc}1+z^{-1} & 0 \\ 0 & 1\end{array}\right]$ which is only NI (and not WSNI nor SSNI).

The following stability theorem here applies to real, rational, proper systems but invokes only the interconnection of NI and WSNI systems. It is the discrete-time counterpart of Theorem 5 in [12].

Theorem 4.1 Let $P: \mathbb{C} \rightarrow \mathbb{C}^{m \times m}$ be a discrete-time, real, rational, proper, NI system with no poles at +1 and -1 , and let $Q: \mathbb{C} \rightarrow \mathbb{C}^{m \times m}$ be a discrete-time, real, rational, proper, WSNI system. Suppose $P(-1) Q(-1)=0$ and $Q(-1) \geq 0$. Then

$$
[P, Q] \text { is internally stable } \Leftrightarrow \quad \bar{\lambda}(P(1) Q(1))<1 .
$$

Proof: The proof trivially follows by applying [12, Theorem 5] or [29, Theorem 1] on the systems $M(s)=P\left(\frac{1+s}{1-s}\right)$ and $N(s)=Q\left(\frac{1+s}{1-s}\right)$ obtained through the bilinear transformation $z=\frac{1+s}{1-s}$.

\section{Concluding remarks}

In this paper we presented a definition of negative imaginary systems for discrete-time systems that hinges entirely on properties of the transfer function matrix and not on a real, rational, proper, finite-dimensional realization. We have drawn a full picture which illustrates the relationship that exists, in the discrete-time, between the notions of positive real and negative imaginary systems, as well as strictly positive real and strictly negative imaginary systems. Indeed, as it happens for the classical theory of positive real systems, even for negative imaginary systems our definitions can be viewed as a single definition referred to different analyticity domains. In fact, we can define a function $G: \mathbb{C} \longrightarrow \mathbb{C}^{m \times m}$ analytic in the open set $\Omega=\{z \in \mathbb{C}:|z|>1\}$, to be is skewimaginary if

- $i\left[G(z)-G(z)^{*}\right] \geq 0$ for all $z \in \Omega$ such that $\mathfrak{I m}\{z\}>0$;

- $i\left[G(z)-G(z)^{*}\right]=0$ for all $z \in \Omega$ such that $\mathfrak{I m}\{z\}=0$;

- $i\left[G(z)-G(z)^{*}\right] \leq 0$ for all $z \in \Omega$ such that $\mathfrak{I m}\{z\}<0$.

Then, it is clear that a function is NI if it is analytic in $\Omega$ and skew-imaginary there.

Finally, we have derived stability analysis results for the interconnections of NI and WSNI systems.

\section{References}

[1] B.D.O. Anderson, and S. Vongpanitlerd. Network Analysis and Synthesis: a Modern Systems Theory Approach. Upper Saddle River, NJ: Prentice Hall, 1973.

[2] B. Bhikkaji, and S. Moheimani. Fast scanning using piezoelectric tube nanopositioners: A negative imaginary approach. Proc. IEEE/ASME Int. Conf. Advanced Intelligent Mechatronics AIM, Singapore, pp. 274-279, July 2009.

[3] B. Bhikkaji, S. O. R. Moheimani, and I. R. Petersen. A negative imaginary approach to modeling and control of a collocated structure. IEEE/ASME Transactions on Mechatronics, 17(4):717-727, 2012.

[4] B. Brogliato, R. Lozano, B. Maschke and O. Egeland. Dissipative Systems Analysis and Control Theory and Applications. 2nd ed. New York: Springer-Verlag, 2007.

[5] O. Brune, The synthesis of a finite two-terminal network whose driving-point impedance is a prescribed function of frequency. Journal of Mathematical Physics, 10: 191-236, 1931. 
[6] C. Cai, and G. Hagen. Stability analysis for a string of coupled stable subsystems with negative imaginary frequency response. IEEE Transactions on Automatic Control, 55(8):1958-1963, Aug 2010.

[7] M. Corless, and R. Shorten. On the Characterization of Strict Positive Realness for General Matrix Transfer Functions. IEEE Transactions on Automatic Control, 55(8):1899-1904, 2010.

[8] A. Ferrante, A. Lanzon, and L. Ntogramatzidis, Foundations of not necessarily rational negative imaginary systems theory: Relations between classes of negative imaginary and positive real systems. IEEE Transactions on Automatic Control, DOI: 10.1109/TAC.2015.2497461. In press.

[9] A. Ferrante, and L. Ntogramatzidis, "Some new results in the theory of negative imaginary systems with symmetric transfer matrix function". Automatica, 49(7): 2138-2144, 2013.

[10] L. Hitz and B. D. O. Anderson, Discrete positive-real functions and their application to system stability, Proceedings of the Institution of Electrical Engineers, 116:153-155, 1969.

[11] H.K. Khalil. Nonlinear Systems. 3rd ed. New Jersey: Prentice Hall, 2002.

[12] A. Lanzon, and I.R. Petersen. Stability robustness of a feedback interconnection of systems with negative imaginary frequency response. IEEE Transactions on Automatic Control, 53(4):10421046, 2008.

[13] A. Lanzon, Z. Song, S. Patra, and I.R. Petersen. A strongly strict negative-imaginary lemma for non-minimal linear systems Communications in Information and Systems, 11(2):139-152, 2011.

[14] J. Xiong, A. Lanzon, and I.R. Petersen. Negative imaginary lemmas for descriptor systems. IEEE Transactions on Automatic Control, 61(2):491-496, 2016.

[15] M.A. Mabrok, A. Kallapur, I.R. Petersen and A. Lanzon. Generalizing negative imaginary systems theory to include free body dynamics: Control of highly resonant structures with free body motion. IEEE Transactions on Automatic Control, 59(10):26922707, 2014.

[16] M.A. Mabrok, A. Kallapur, I.R. Petersen and A. Lanzon. Spectral conditions for negative imaginary systems with applications to nanopositioning. IEEE/ASME Transactions on Mechatronics, 19(3):895903, 2014.

[17] I. A. Mahmood, S. O. R. Moheimani, and B. Bhikkaji. A new scanning method for fast atomic force microscopy. IEEE Transactions on Nanotechnology, 10(2):203-216, 2011.

[18] M. Opmeer. Infinite-dimensional negative imaginary systems. IEEE Transactions on Automatic Control, 56(12):2973-2976, 2011.

[19] S. Patra, and A. Lanzon. Stability analysis of interconnected systems with 'mixed' negative-imaginary and small-gain properties. IEEE Transactions on Automatic Control, 56(6):1395-1400, 2011.

[20] I.R. Petersen, and A. Lanzon. Feedback control of negative-imaginary systems. IEEE Control Systems Magazine, 30(5):54-72, 2010.

[21] K. Premaratne and E.I. Jury. Discrete-time Positive-Real Lemma revisited: the discrete-time counterpart of the Kalman-Yakubovitch Lemma IEEE Transactions on Circuits and Systems I: Fundamental Theory and Applications, 41(11):747-750, 1994.

[22] W. Rudin. Real and Complex Analysis. Third Edition. McGrow-Hill, 1987.

[23] Z. Song, A. Lanzon, S Patra, I.R. Petersen. A negative-imaginary lemma without minimality assumptions and robust state-feedback synthesis for uncertain negative-imaginary systems Systems and Control Letters, 61(12):1269-1276, 2012.

[24] G. Tao, and P.A. Ioannou. Necessary and sufficient conditions for strictly positive real matrices. IEE Proceedings, 137(5):360-366, 1990.
[25] A. van der Schaft. Positive feedback interconnection of Hamiltonian systems. Proceedings of the 50th IEEE Conference on Decision and Control and European Control Conference, Orlando, FL, USA, Dec 2011.

[26] J. Wang, A. Lanzon, and I.R. Petersen. Robust output feedback consensus for networked negative-imaginary systems. IEEE Transactions on Automatic Control, 60(9):2547-2552, 2015

[27] J. Wang, A. Lanzon, and I.R. Petersen. Robust cooperative control of multiple heterogeneous negative-imaginary systems. Automatica, 61:64-72, 2015.

[28] C. Xiao and B. J. Hills, Generalizations and new proof of the discrete-time Positive Real Lemma and Bounded Real Lemma, IEEE Transactions on Circuits and Systems - I, 46(6):740-743, 1999.

[29] J. Xiong, I.R. Petersen, and A. Lanzon. A negative imaginary lemma and the stability of interconnections of linear negative imaginary systems. IEEE Transactions on Automatic Control, 55(10):23422347, 2010.

[30] J. Xiong, I.R. Petersen, and A. Lanzon. On lossless negative imaginary systems. Automatica, 48(6): 1213-1217, 2012.

[31] M. Zorzi. Rational approximations of spectral densities based on the Alpha divergence, Mathematics of Control, Signals, and Systems. Vol. 26(2): 259-278, 2014.

[32] M. Zorzi, and A. Chiuso. A Bayesian approach to sparse plus low rank network identification. 54th IEEE Conference on Decision and Control, pp. 7386-7391, 2015.

[33] M. Zorzi, and R. Sepulchre. AR Identification of Latent-Variable Graphical Models. IEEE Transactions on Automatic Control. To appear (doi: 10.1109/TAC.2015.2491678; available on-line at http://ieeexplore.ieee.org/stamp/stamp.jsp?tp=\&arnumber $=7308026 \&$ isnumber=4601496), 2016. 